\title{
Кондаков А.С. \\ Тепловая диагностика трения в системе полимерных подшипни-ков на общем валу по температурным данным
}

Институт проблем нефти и газа СО РАН

(Россия, Якутск)

doi: $10.18411 / l j-30-11-2017-54$

idsp: 000001:lj-30-11-2017-54

\section{Аннотация}

Предложен метод тепловой диагностики трения, позволяющий определить мощности трения в системе полимерных подшипников на общем валу с переменным поперечным сечением на основе решения граничной обратной задачи теплообмена. Эффективность метода подтверждаются проведением вычислительных экспериментов с возмущёнными температурными данными.

Ключевые слова: узел трения, система полимерных подшипников, температура, мощность трения, обратная задача, регуляризация, вычислительный эксперимент.

1. Введение. Предлагаемый метод тепловой диагностики узлов трения основан на следующих фактах. Во-первых, практически вся механическая энергия, затрачиваемая на трение, трансформируется в тепловую [1]. Во-вторых, существующие методы непосредственного замера мощности трения предусматривают использование упругих элементов, установка которых, даже в условиях стендовых испытаний узлов трения, крайне затруднительно. По этой причине, в условиях эксплуатации и при проведении стендовых испытаний не всегда удается получить данные о потерях на трение, что существенно затрудняет определение основных триботехнических параметров, необходимых для прогнозирования работоспособности и оценки технического состояния узлов трения. Это приводит к необходимости определять работу, затраченную на трение, по замерам других величин, достаточно хорошо коррелирующих с искомым. С точки зрения доступности измерений, наиболее выгодным является температурная информация, не требующая для измерения сложного и громоздкого оборудования. Температура более доступна для непосредственного измерения, включая самые неблагоприятные случаи.

Регистрация температуры в окрестности зоны трения, построение математической тепловой модели, адекватной процессу теплообмена в сопряжении, и решение соответствующей граничной обратной задачи позволяет восстановить теплоту, выделившуюся в результате трения. И, если известна часть энергии трения, которая трансформируется в теплоту, появляется принципиальная возможность количественной оценки работы, затраченной на трение [2]. Поэтому необходимо решать обратную задачу определения функции тепловыделения по замерам температуры внутри одного из элементов трибосистемы с учетом пространственного распределения температуры в нём. Для решения трехмерной граничной обратной задачи необходимо задавать замеры температуры на некоторой поверхности внутри одного из элементов сопряжения, что практически невыполнимо из-за возможного нарушения целостности материала и искажения температурного поля. Такое положение приводит к необходимости использования таких математических тепловых моделей, которые достаточно адекватно описывают реальное температурное поле в сопряжении и значительно сокращают количество точек замера температуры, необходимых для восстановления функции тепловыделения и соответственно мощности трения.

Для решения приведённой ниже обратной задачи теплообмена используется метод итерационной регуляризации на основе градиентных методов минимизации функционала невязки рассчитанных температур от заданных [3].

Рассмотрим систему полимерных подшипников скольжения (рис.1), для которых характерны многие особенности контактного взаимодействия цилиндрических сопряжений. Скольжение происходит по поверхности контакта полимерных втулок с 
металлическим валом. Углы контакта $\varphi_{0 k}$ известны и неизменны в течение всего времени испытаний tm. Примем следующие основные допущения: нагрузки равномерно распределены по длине подшипников; теплообмен с торцов подшипников и корпусов пренебрежимо мал; распределение температуры по длине подшипников однородно; скорость вращения вала достаточно высока, что позволяет принять тепловой поток на границе равномерно распределенным по окружности вала и температурное поле в поперечном сечении вала однородным.

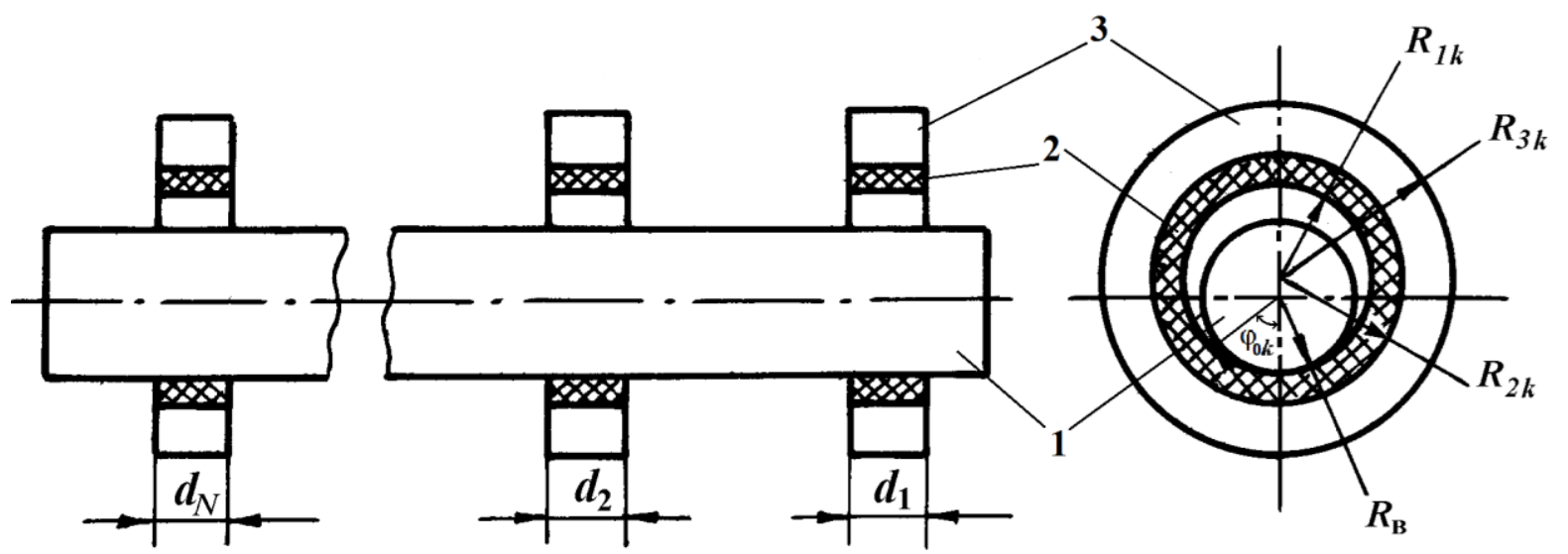

Рис. 1. Схема системы подшипников на общем валу. 1-вал, 2 - полимерная втулка, 3 -корпус

Тогда нестационарные температурные поля для вала, в общем случае с переменным поперечным сечением, представляется одномерным уравнением, а для элементов подшипников - двумерными уравнениями в цилиндрических координатах $(r, \varphi)$. При принятых допущениях напишем упрощенную квазитрёхмерную математическую тепловую модель для системы подшипников [4]. В зонах контакта $A_{k}$ вала с подшипниками $\left(|\varphi| \leq \varphi_{0 k}, k=1,2, \ldots, N\right)$ происходит тепловыделение с мощностью $Q_{k}(t)$. Уравнение теплопроводности для вала записывается в виде:

$$
\begin{gathered}
S(z) C_{\mathrm{B}}(U) \frac{\partial U}{\partial t}=\frac{\partial}{\partial z}\left[S(z) \lambda_{\mathrm{B}}(U) \frac{\partial U}{\partial z}\right]-P(z)\left[1-\Theta_{k}(z)\right] \alpha_{\mathrm{B}}\left(U-T_{\mathrm{cp}}\right)+ \\
+\Theta_{k}(z) \cdot \sum_{k=1}^{N}\left[\frac{Q_{k}(t)}{d_{k}}+\left.2 R_{1 k} \int_{0}^{\varphi_{\mathrm{o}}} \lambda_{k}\left(T_{k}\right) \frac{\partial T_{k}}{\partial r}\right|_{r=R_{1 k}} d \varphi\right] \\
U=U(z, t), 0<z<L, \quad 0<t \leq t_{m},
\end{gathered}
$$

где $\mathrm{S}(\mathrm{z}), \mathrm{P}(\mathrm{z})$ - функции площади и периметра поперечного сечения вала; tm- время испытания; $\alpha \mathrm{B}$ - коэффициент теплообмена вала с окружающей средой; Тср- температура окружающей среды; L - длина вала; $\Theta_{k}(z)$ - характеристическая функция множества точек $A_{k}$ контакта $\mathrm{k}$ - го подшипника с валом: $\Theta_{k}(z)$ равно 1 при z принадлежащем $A_{k}$ равно 0 в противном случае.

Уравнение теплопроводности для каждого подшипника имеет вид:

$$
\begin{aligned}
C_{k}\left(T_{k}\right) \frac{\partial T_{k}}{\partial t} & =\frac{1}{r} \frac{\partial}{\partial r}\left(r \lambda_{k}\left(T_{k}\right) \frac{\partial T_{k}}{\partial r}\right)+\frac{1}{r^{2}} \frac{\partial}{\partial \varphi}\left(\lambda_{k}\left(T_{k}\right) \frac{\partial T_{k}}{\partial \varphi}\right), \\
0 & <t \leq t_{m}, \quad R_{1 k}<r<R_{3 k}, \quad 0<\varphi<\pi, k=\overline{1, N} .
\end{aligned}
$$


В силу высокой теплопроводности вала и малой длины контакта распределение температуры по длине втулок можно принять однородным и равным осредненному значению температуры вала в контактирующей зоне

$$
T_{k}\left(R_{1 k}, \varphi, t\right)=\frac{1}{d_{k}} \int_{A_{k}} U(z, t) d z, \quad|\varphi| \leq \varphi_{\mathrm{o} k}, \quad k=\overline{1, N} .
$$

Из уравнения (1) видно, что на вал в точках его контакта с втулками действуют источники тепла, равные разности возникающих при трении мощностей тепловыделения $Q_{k}(t), \mathrm{k}=1,2, \ldots, \mathrm{N}$ и долей мощностей, отводимых во втулки. Распределение температуры в вале по длине контакта с втулками будет неоднородным.

Уравнения (1) и (2) дополняется начальными распределениями температур, граничными условиями третьего рода на свободных от контакта с валом поверхностях подшипников и граничными условиями первого рода на концах вала.

Теперь сформулируем задачу восстановления мощностей тепловыделений $Q_{k}(t)$, $\mathrm{k}=1,2, \ldots, \mathrm{N}$ по замеру температуры в одной или в нескольких точках каждой втулки на известном расстоянии от поверхности трения.

Пусть во втулках на фиксированных радиусах Rk заданы замеры температуры $f_{k}\left(\varphi_{k j}, t\right)$ по окружности в точках с угловыми координатами $\varphi_{k j}, j=1,2, \ldots, M_{k}$

Обратная задача восстановления функций мощностей тепловыделений $Q_{k}(t)$ по температурной данным $f_{k}\left(\varphi_{k j}, t\right)$ сводится к минимизации функционала невязки

$$
J\left[Q_{1}, Q_{2}, \ldots, Q_{N}\right]=\frac{1}{2} \sum_{k=1}^{N} \sum_{j=1}^{M_{k}} \int_{0}^{t_{m}}\left[T\left(R_{k}, \varphi_{k j}, t\right)-f_{k}\left(\varphi_{k j}, t\right)\right]^{2} d t,
$$

характеризующего меру уклонения рассчитанных температур от заданных.

Компоненты градиентов функционала (5) для каждого подшипника определяются решением соответствующей сопряжённой задачи. Все начально краевые дифференциальные задачи решаются численно методом конечных разностей.

Были проведены вычислительные эксперименты для проверки эффективности разработанного метода решения обратной задачи. Для чего задавались модельные функции мощностей тепловыделения и решались прямые задачи. Результаты вычисленных температур в фиксированных точках запоминались и использовались как температурные данные для решения обратной задачи определения мощностей тепловыделений $Q_{k}(t)$ как искомых. В дальнейшем эти задаваемые мощности и температурные данные будем называть модельными. Для лучшего контроля работоспособности алгоритма вычислительной программы была принята симметричная относительно среднего подшипника схема, для которой на крайних подшипниках задавались одинаковые функции мощностей тепловыделений.

Модельные задачи решались для системы из трёх подшипников на общем валу с радиусом 0,0145 м и длиной 0,46 м с симметрией относительно среднего подшипника. Геометрические размеры втулок и корпусов брались одинаковыми и взяты следующими:

$$
R_{1 k}=0,015 ; \quad R_{2 k}=0,018 ; \quad R_{3 k}=0,036 \quad \text { м, } k=1,2,3 .
$$

Расстояния между подшипниками равны 0,1 м. Длины подшипников одинаковы: $d_{k}=0,02 \mathrm{M}, k=1,2,3$. Полууголы контактов втулок с валом тоже одинаковы: $\varphi_{0 k}=14^{\circ}, k=1,2,3$. На концах вала заданы одинаковые условия первого рода, что должно обеспечивать симметричность температурных полей относительно середины системы при задании одинаковых функций тепловыделения на крайних подшипниках скольжения. 
Материалом для вала и корпусов подшипников служит сталь, а для втулок наполненный фторопласт. Зависимости теплофизических свойств от температуры для наполненного фторопласта имеют вид:

$$
\begin{aligned}
& \lambda_{k}=0,07(T-100) / 150+0,35 \quad\left(\frac{B m}{\mu \cdot{ }^{\circ} C}\right), \\
& C_{k}=\left[6 \cdot 10^{-3}(T-30)+3\right] \cdot 10^{6} \quad\left(\frac{\text { Дж }}{\mu^{3} \cdot{ }^{\circ} C}\right), \quad k=1,2,3 .
\end{aligned}
$$

Для стали:

$$
\begin{aligned}
& \lambda_{\mathrm{B}}=30,5(T-100) / 150+55,5\left(\frac{B m}{\mathcal{M} \cdot{ }^{\circ} \mathrm{C}}\right), \\
& C_{\mathrm{B}}=\left[1,2 \cdot 10^{-3}(T-30)+3,7\right] \cdot 10^{6}\left(\frac{\text { Джс }}{\mathrm{M}^{3} \cdot{ }^{\circ} \mathrm{C}}\right) .
\end{aligned}
$$

На рис.2(a) приведены результаты расчёта восстановления мощностей теплообразования с точно заданными температурными данными. С построенных графиков функций видно, что восстановления искомых мощностей достаточно приближённо точны с наибольшими отклонениями в точках излома модельных функций. Надо заметить, что незначительные погрешности восстановленных функций от точных модельных можно объяснить приближённостью принятой тепловой модели.

Наибольший интерес представляет собой чувствительность предложенного метода к погрешностям температурных данных, т.е. устойчивость алгоритма к возмущениям входных данных. Возмущения входных температурных данных имитировались выражениями

$$
\overline{f_{k}}\left(\varphi_{k j}, t\right)=f_{k}\left(\varphi_{k j}, t\right)+2 \Delta(\sigma(t)-0.5), k=1,2,3 ; 0 \leq t \leq t_{m},
$$

где $\sigma(\mathrm{t})$ - нормально распределенная на отрезке $[0,1]$ случайная функция; $\Delta$ - уровень погрешности. Теперь при решении обратной задачи вместе точных температурных данных использовались возмущённые.

На рис.2(б, в) приведены результаты расчёта восстановления при различных значениях погрешности. Приведённые графики на рисунках показывают удовлетворительную устойчивость метода к погрешностям температурных данных, уровень которых выше чем погрешности традиционных измерительных приборов.

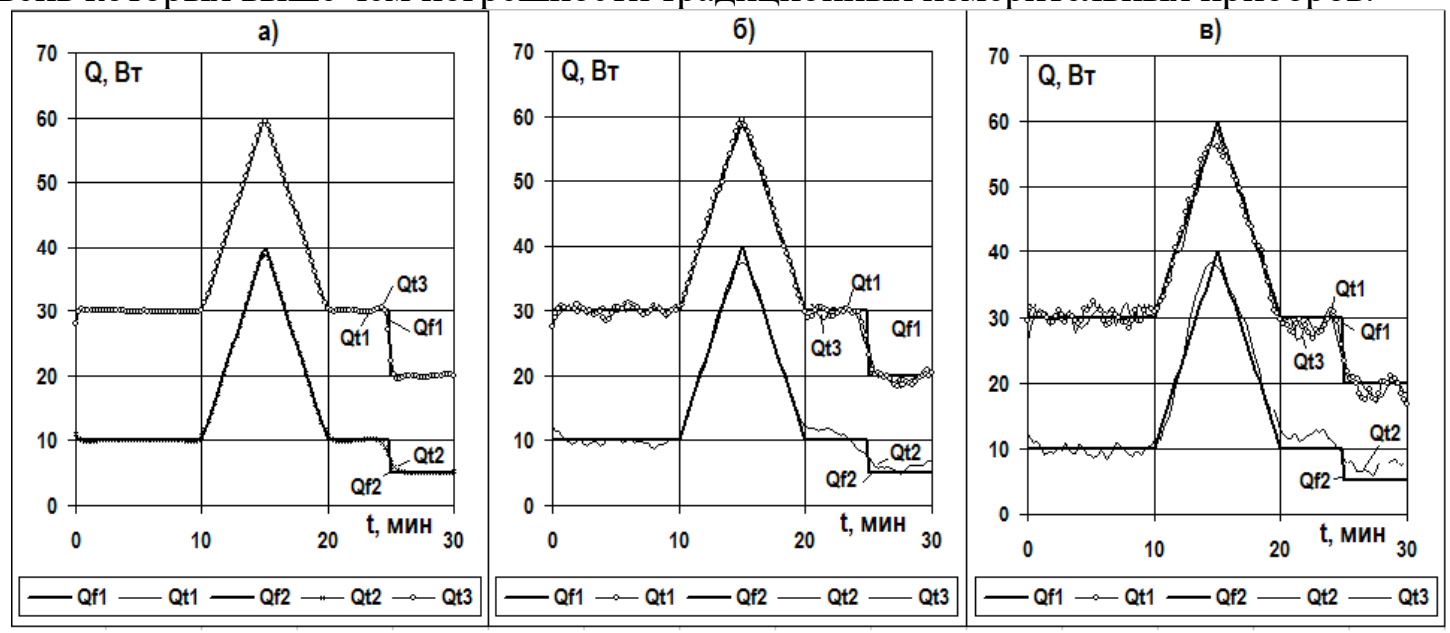

Рис.2. Восстановление мощностей тепловыделения в системе трех подшипников на общем валу: а) -c точными температурными данными; б) и в) - с возмущенными температурными данными соответственно с уровнями возмущения равньми 1 и 3 градусов Цельсия. Qf1-Qf3 (Qf1=Qf3)-модельнье мощчности, Qt1-Qt3 - восстановленные мощчности. 
Проведённые вычислительные эксперименты показывают практическую пригодность предложенной методики для определения мощностей трения в реальных узлах трения с системой подшипников на одном валу.

\section{$* * *$}

1. Костецкий Б.И., Линник Ю.И. Энергетический баланс при внешнем трении металлов // ДАН СССР. 1968. - Т. 183. - № 5. - С. 42-46.

2. Старостин Н.П., Тихонов А.Г., Моров В.А., Кондаков А.С. Расчет триботехнических параметров в опорах скольжения. - Якутск. - 1999. - 274 с.

3. Алифанов О.М., Артюхин Е.А., Румянцев С.В. Экстремальные методы решения некорректных задач. Москва: Наука, 1988. - 288 с.

4. Старостин Н.П., Кондаков А.С. Нелинейная математическая модель теплового режима в системе подшипников скольжения на общем валу // Труды II Евразийского симпозиума по проблемам прочности материалов и машин для регионов холодного климата. Часть IV. Полимерные композиционные материалы и изделия для эксплуатации в условиях холодного климата. - Якутск, 2004. - C. 164- 175 .

\section{Котов А.Д., Михайловская А.В., Ситкина М.Н., Борисов А.А. Сверхпластичность сплавов системы Al-Zn-Mg-Cu-Ni-Zr с разным содержанием Znи Mg}

НИТУ «МИСиС» (Россия, Москва)

doi: $10.18411 / l j-30-11-2017-55$

idsp: 000001:lj-30-11-2017-55

\section{Аннотация}

В настоящей работе были исследованы сплавы на основе системы $\mathrm{Al}-\mathrm{Zn}-\mathrm{Mg}-\mathrm{Cu}$ $\mathrm{Ni}-\mathrm{Zr}$, которые послетермо деформационной обработки имеют структуру естественного композита с бимодальным распределением частиц по размерам. Показано, что для формирования микрозеренной структуры и проявления сверхпластичности помимо бимодального распределения частиц по размерам также обязательно наличие сильнолегированного твердого раствора, так как в случае его малой легированности в сплавах исследуемой системы затруднена рекристаллизацияво время нагрева, а также при начальной стадии сверхпластической деформации. Увеличение содержания как $\mathrm{Zn}$, так и $\mathrm{Mg}$ в алюминиевом твердом растворе способствует формированию более мелкого зерна, снижению напряжения течения, увеличению показателя скоростной чувствительности $\mathrm{m}$ и росту значений относительного удлинения.

Ключевые слова: алюминиевые сплавы, сверхпластичность, мелкозернистая структура, напряжение течения, относительное удлинение

\section{Введение}

Высокопрочные сплавы на основе системы $\mathrm{Al}-\mathrm{Zn}-\mathrm{Mg}-\mathrm{Cu}$ являются перспективными с точки зрения механических свойств при комнатной температуре, однако наиболее известные промышленные сплавы данной системы проявляют сверхпластичность только при довольно низких скоростях деформации 10-4 с-1[1,2]. В настоящее время в работах по сверхпластичности термически упрочняемых алюминиевых сплавов в основном реализованы способы получения микрозеренной структуры, основанные налегировании сплавов элементами, образующими в структуре дисперсные частицы (Sc, Zr, Mn, Cr),сдерживающие рост зерен [3-5], или измельчении зерна путем применения интенсивной деформации [6-8]. По составу исследуемые сплавы (по $\mathrm{Zn}, \mathrm{Mg}$, $\mathrm{Cu})$ в основном соответствуют применяемым в промышленности сплавам, имеющим высокие прочностные характеристики. При этом влияние легированности твердого раствора указанными элементами на размер зерна и показатели сверхпластичности освещено слабо. 